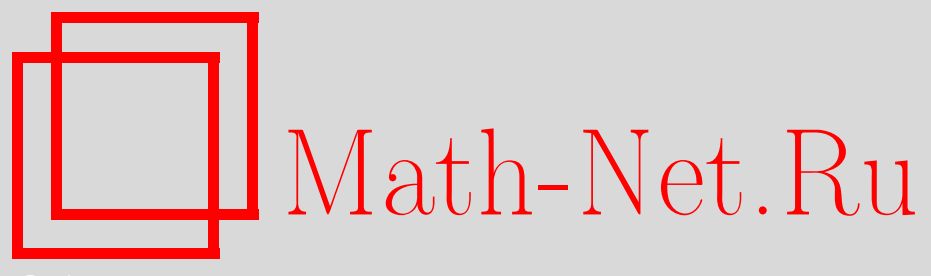

М. Е. Широков, Об одном свойстве выходной энтропии положительного отображения между пространствами ядерных операторов, Матем. заметки, 2010, том 87, выпуск 3, 477-479

DOI: https://doi.org/10.4213/mzm8680

Использование Общероссийского математического портала Math-Net.Ru подразумевает, что вы прочитали и согласны с пользовательским соглашением http://www . mathnet.ru/rus/agreement

Параметры загрузки:

IP : 54.174 .149 .18

26 апреля 2023 г., 13:18:25

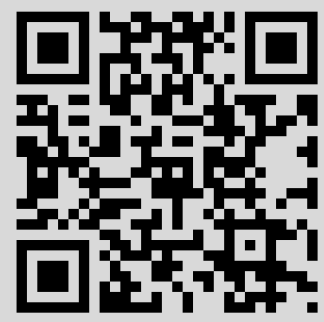




\section{Об одном свойстве выходной энтропии положительного отображения между пространствами ядерных операторов}

\section{М. Е. Широков}

Введение. При исследовании информационных характеристик квантовых каналов связи (линейных вполне положительных сохраняющих след отображений между пространствами ядерных операторов) важную роль играет выходная энтропия фон Неймана этого канала, рассматриваемая как функция на множестве входных состояний (положительных операторов с единичным следом) этого канала [1]. В квантовой статистике также используется понятие квантовой операции (линейного вполне положительного неувеличивающего след отображения) и его выходной энтропии [1].

Выходная энтропия квантовых каналов и операций между конечномерными квантовыми системами является непрерывной вогнутой функцией на компактном множестве входных квантовых состояний, однако ее свойства радикально меняются при переходе к бесконечномерному случаю. Это связано с тем, что энтропия фон Неймана является разрывной функцией на множестве состояний бесконечномерной квантовой системы, принимающей значение $+\infty$ везде, за исключением подмножества первой категории [2]. Именно с этим обстоятельством связаны как трудности анализа квантовых систем и каналов бесконечной размерности, так и специфические свойства их характеристик (например, разрывность пропускной способности как функции канала и т.п.).

Тем не менее, существуют нетривиальные бесконечномерные квантовые каналы и операции, выходная энтропия которых непрерывна, что существенно облегчает анализ их информационных свойств [3]. Цель данного сообщения - показать, что для доказательства непрерывности выходной энтропии положительного линейного отображения между пространствами ядерных операторов (в частности, квантового канала или квантовой операции) на множестве входных состояний достаточно показать ее конечность на этом множестве.

Определения и обозначения. Пусть $\mathscr{H}$ - сепарабельное гильбертово пространство, $\mathfrak{B}(\mathscr{H})$ - алгебра всех ограниченных операторов в $\mathscr{H}$ с операторной нормой $\|\cdot\|$, $\mathfrak{T}(\mathscr{H})$ - сепарабельное банахово пространство ядерных операторов в $\mathscr{H}$ со следовой нормой $\|\cdot\|_{1}=\operatorname{Tr}|\cdot|$ с конусом $\mathfrak{T}_{+}(\mathscr{H})$ положительных ядерных операторов, содержащим замкнутое выпуклое подмножество $\mathfrak{S}(\mathscr{H})$ положительных ядерных операторов с единичным следом. Операторы из $\mathfrak{S}(\mathscr{H})$ будем обозначать символами $\rho, \sigma, \ldots$ и называть состояниями, поскольку каждый такой оператор $\rho$ определяет нормальное состояние $A \mapsto \operatorname{Tr} A \rho$ на алгебре $\mathfrak{B}(\mathscr{H})[1]$.

Энтропия фон Неймана $H(\rho)=-\operatorname{Tr} \rho \log \rho$ состояния $\rho \in \mathfrak{S}(\mathscr{H})$ имеет следующее естественное продолжение на конус $\mathfrak{T}_{+}(\mathscr{H})($ см. [4])

$$
H(A)=\operatorname{Tr} A H\left(\frac{A}{\operatorname{Tr} A}\right)=\operatorname{Tr} \eta(A)-\eta(\operatorname{Tr} A), \quad A \in \mathfrak{T}_{+}(\mathscr{H}), \quad \eta(x)=-x \log x .
$$

Из неотрицательности, вогнутости и полунепрерывности снизу энтропии фон Неймана на множестве $\mathfrak{S}(\mathscr{H})$ следуют соответствующие свойства функции $A \mapsto H(A)$ на множестве $\mathfrak{T}_{+}(\mathscr{H})$, которую будем называть квантовой энтропией. Функцию $\left\{x_{i}\right\} \mapsto H\left(\left\{x_{i}\right\}\right)=$

Работа выполнена при поддержке АВЦП "Развитие научного потенциала высшей школы" (грант № 2.1.1/500), ФЦП "Научные и научно-педагогические кадры инновационной России" (грант № HK-13П/4) и Российского фонда фундаментальных исследований (грант № 09-01-00424-a). 
$\sum_{i} \eta\left(x_{i}\right)-\eta\left(\sum_{i} x_{i}\right)$ на положительном конусе банахова пространства $l_{1}$ суммируемых последовательностей, совпадающую с энтропией Шеннона на множестве всех распределений вероятностей $\mathfrak{P}_{+\infty}$, будем называть классической энтропией.

Основной результат. Пусть $\Phi: \mathfrak{T}(\mathscr{H}) \rightarrow \mathfrak{T}\left(\mathscr{H}^{\prime}\right)$ - положительное линейное ограниченное отображение. Выходная энтропия $H \circ \Phi$ этого отображения - вогнутая полунепрерывная снизу функция на $\mathfrak{S}(\mathscr{H}) \subset \mathfrak{T}(\mathscr{H})$ со значениями в $[0,+\infty]$. Следующая теорема показывает, что эта функция не может быть разрывной, если она принимает только конечные значения.

Теорема 1. Пусть $\Phi$ - линейное положителъное ограниченное отображение из $\mathfrak{T}(\mathscr{H})$ в $\mathfrak{T}\left(\mathscr{H}^{\prime}\right)$. Следующие свойства равносильны:

(i) функиия $\rho \mapsto H(\Phi(\rho))$ конечна на множестве $\mathfrak{S}(\mathscr{H})$;

(ii) функиия $\rho \mapsto H(\Phi(\rho))$ непрерывна и ограничена на множестве $\mathfrak{S}(\mathscr{H})$;

(iii) существует ортонормированный базис $\{|i\rangle\}_{i=1}^{+\infty}$ пространства $\mathscr{H}^{\prime}$ такой, что функиия $\rho \mapsto H\left(\{\langle i|\Phi(\rho)| i\rangle\}_{i=1}^{+\infty}\right)$ непрерывна и ограничена на множестве $\mathfrak{S}(\mathscr{H})$

(iv) существует ортонормированный базис $\{|i\rangle\}_{i=1}^{+\infty}$ пространства $\mathscr{H}^{\prime}$ и последовательность неотрицательных чисел $\left\{h_{i}\right\}_{i=1}^{+\infty}$ такие, что

$$
\left\|\sum_{i=1}^{+\infty} h_{i} \Phi^{*}(|i\rangle\langle i|)\right\|<+\infty \quad u \quad \sum_{i=1}^{+\infty} e^{-h_{i}}<+\infty,
$$

где $\Phi^{*}$ - дуальное $\kappa \Phi$ отображение из $\mathfrak{B}\left(\mathscr{H}^{\prime}\right)$ в $\mathfrak{B}(\mathscr{H}){ }^{1}$

В доказательстве данной теоремы используются некоторые специальные свойства энтропии фон Неймана (см. [2], [4]), а также результаты, связанные с понятием $\chi$-емкости множества квантовых состояний, полученные в [5]. Это доказательство показывает, что роль множества $\mathfrak{S}(\mathscr{H})$ в теореме 1 может играть любое ограниченное выпуклое подмножество $\mathscr{A}$ конуса $\mathfrak{T}_{+}(\mathscr{H})$ такое, что из $\sup _{A \in \mathscr{A}} \lim _{n \rightarrow+\infty} \operatorname{Tr} A B_{n}<+\infty$ следует $\sup _{n}\left\|B_{n}\right\|<+\infty$ для любой возрастающей последовательности $\left\{B_{n}\right\}$ положительных операторов из $\mathfrak{B}(\mathscr{H})$.

ЗАмечание 1. Теорема 1 не утверждает, что из свойства (i), т.е. конечности квантовой энтропии на множестве $\Phi(\mathfrak{S}(\mathscr{H}))$, следует ее непрерывность на этом множестве, поскольку из непрерывности функции $\rho \mapsto H(\Phi(\rho))$ на некомпактном множестве $\mathfrak{S}(\mathscr{H})$ не следует непрерывность функции $A \mapsto H(A)$ на множестве $\Phi(\mathfrak{S}(\mathscr{H}))$, что подтверждает следующий пример.

Пусть $\mathscr{A}$ - такое выпуклое замкнутое подмножество множества $\mathfrak{S}\left(\mathscr{H}^{\prime}\right)$, на котором энтропия фон Неймана разрывна, но ограничена (см. примеры в [5]). Пусть $\left\{\sigma_{n}\right\}_{n=1}^{+\infty}-$ последовательность состояний из $\mathscr{A}$, сходящаяся к состоянию $\sigma_{0}$ из $\mathscr{A}$, такая, что $\lim _{n \rightarrow+\infty} H\left(\sigma_{n}\right) \neq H\left(\sigma_{0}\right)$. Рассмотрим отображение $\Phi: \rho \mapsto \sum_{n=0}^{+\infty}\langle n|\rho| n\rangle \sigma_{n}$, где $\{|n\rangle\}_{n=0}^{+\infty}-$ некоторый ортонормированный базис в пространстве $\mathscr{H}^{\prime}$. В силу теоремы 1 функция $\rho \mapsto H(\Phi(\rho))$ непрерывна на множестве $\mathfrak{S}(\mathscr{H})$, но функция $A \mapsto H(A)$ разрывна на множестве $\Phi(\mathfrak{S}(\mathscr{H}))$, содержащем последовательность $\left\{\sigma_{n}\right\}_{n=1}^{+\infty}$.

Непрерывность функции $\rho \mapsto H(\Phi(\rho))$ на множестве $\mathfrak{S}(\mathscr{H})$ равносильна непрерывности функции $A \mapsto H(A)$ на любом множестве вида $\Phi(\mathscr{C})$, где $\mathscr{C}$ - компактное подмножество множества $\mathfrak{S}(\mathscr{H})$.

ЗАмечАниЕ 2. Главное утверждение теоремы 1 (импликация (i) $\Rightarrow$ (ii)) основано на особых свойствах энтропии фон Неймана, его нельзя доказать, используя только такие общие свойства функций энтропийного типа, как вогнутость, полунепрерывность снизу и т.п. Простейший пример, подтверждающий данное замечание, - выходная энтропия Реньи порядка $p=0$ отображения $\Phi$, т.е. функция $\rho \mapsto \log \operatorname{rank}(\Phi(\rho))$.

\footnotetext{
${ }^{1}$ Отображение $\Phi^{*}$ определяется соотношением $\operatorname{Tr} \Phi^{*}(A) \rho=\operatorname{Tr} A \Phi(\rho), \rho \in \mathfrak{S}(\mathscr{H})$.
} 
ЗАмечание 3. Свойство (iii) в теореме 1 является формальным усилением свойства (ii), поскольку непрерывность (соответственно, ограниченность) квантовой энтропии на множестве $\mathscr{A} \subset \mathfrak{T}_{+}(\mathscr{H})$ всегда следует из непрерывности (соответственно, ограниченности) классической энтропии на множестве $\left\{\{\langle i|A| i\rangle\}_{i=1}^{+\infty} \mid A \in \mathscr{A}\right\}$ для хотя бы одного базиса $\{|i\rangle\}_{i=1}^{+\infty}$ пространства $\mathscr{H}[5 ;$ предложение 5], а обратное утверждение не верно.

Свойство (iv) в теореме 1 можно рассматривать как критерий непрерывности выходной энтропии положительного отображения в терминах дуального отображения.

Пример 1. Простейшее линейное положительное отображение из $\mathfrak{T}(\mathscr{H})$ в $\mathfrak{T}\left(\mathscr{H}^{\prime}\right)$ имеет вид $\Phi_{V}(\cdot)=V(\cdot) V^{*}$, где $V$ - ограниченный линейный оператор из $\mathscr{H}$ в $\mathscr{H}^{\prime}$. Используя импликацию (iv) $\Rightarrow$ (ii) в теореме 1 и результаты из [5], можно показать, что функция $\rho \mapsto H\left(V \rho V^{*}\right)$ непрерывна на множестве $\mathfrak{S}(\mathscr{H})$ тогда и только тогда, когда оператор $V$ компактен и последовательность его сингулярных чисел $\left\{s_{i}\right\}$ (собственных значений оператора $V^{*} V$ ) такова, что $\sum_{i=1}^{+\infty} e^{-\lambda / s_{i}}<+\infty$ при некотором $\lambda>0$ (считаем, что $\left.e^{-\lambda / 0}=0\right)$.

Используя теорему 1 можно существенно упростить доказательства непрерывности выходной энтропии квантовых каналов, рассмотренных в [3], а также получить общие условия непрерывности выходной энтропии квантовых каналов и операций в терминах их представления Крауса [1].

В заключение приведем коммутативный вариант теоремы 1 , который может быть полезен при исследовании выходной энтропии Шеннона марковских и субмарковских операторов.

СлеДСтвиЕ 1. Пусть $\left\|\phi_{i j}\right\|$ - матрица положительного линейного ограниченного преобразования в пространстве $l_{1}$. Следующие свойства равносильны:

(i) функция $\left\{\pi_{i}\right\} \mapsto H\left(\left\{\sum_{j} \phi_{i j} \pi_{j}\right\}_{i=1}^{+\infty}\right)$ конечна на множестве $\mathfrak{P}_{+\infty}$;

(ii) бункция $\left\{\pi_{i}\right\} \mapsto H\left(\left\{\sum_{j} \phi_{i j} \pi_{j}\right\}_{i=1}^{+\infty}\right)$ непрерывна и ограничена на множестве $\mathfrak{P}_{+\infty}$;

(iii) существует последовательность неотрицательных чисел $\left\{h_{i}\right\}_{i=1}^{+\infty}$ такая, что $\sup _{j} \sum_{i=1}^{+\infty} h_{i} \phi_{i j}<+\infty u \sum_{i=1}^{+\infty} e^{-h_{i}}<+\infty$.

Автор выражает благодарность участникам семинара "Квантовая вероятность, статистика, информация" под руководством профессора А. С. Холево за внимание к работе и полезные замечания.

\section{СПИСОК ЦИТИРОВАННОЙ ЛИТЕРАТУРЫ}

[1] А. С. Холево, Статистическая структура квантовой теории, ИКИ, М.-Ижевск, 2003. [2] A. Wehrl, Rev. Modern Phys., 50:2 (1978), 221-260. [3] М. Е. Широков, ТВП, 53:4 (2008), 732-750. [4] G. Lindblad, Comm. Math. Phys., 39:2 (1974), 111-119. [5] М. Е. Широков, Изв. РАН. Сер. матем., 70:6 (2006), 193-222.

\section{М. Е. Широков}

Поступило

Математический институт им. В. А. Стеклова РАН

20.10.2009

E-mail: msh@mi.ras.ru 